\title{
Construcción y Validación del Inventario de Respuestas Emocionales Negativas y Perturbadoras en Contextos de Pandemias (IREN-35) en población mexicana Construction and Validation of the Inventory of Negative and Disturbing Emotional Responses in Pandemic Contexts (IREN-35) in the Mexican population
}

\author{
Rogelio Flores-Morales ${ }^{1}$ (i) http://orcid.org/0000-0002-3424-2628 \\ Lucy María Reidil-Martínez ${ }^{1}$ (D) http://orcid.org/0000-0003-4163-2293 \\ Adriana Karime Adame-Rivas ${ }^{1}$ (1) http://orcid.org/0000-0002-6109-8764 \\ Verónica Reyes-Pérez ${ }^{2}$ (I) http://orcid.org/0000-0002-2312-8635 \\ ${ }^{1}$ Universidad Nacional Autónoma de México: rogelio.flores@psicologia.unam.mx \\ ${ }^{2}$ Universidad de Guanajuato \\ (c) Universidad De La Salle Bajío (México)
}

Palabras clave: inventario; instrumentos psicométricos; emociones negativas; pandemia; COVID-19; coronavirus; propiedades psicométricas; salud mental; psicología; emociones; población; México; virus; confinamiento; aislamiento; salud; medición; SARS-CoV-2

Keywords: inventory; psychometric instruments; negative emotions; pandemics; COVID-19; coronavirus; psychometric properties; mental health; psychology; emotions; population; Mexico; virus; confinement; isolation; health; measurement; SARS-CoV-2

Recibido en: 16 - 10 - 2020 / Aceptado en: $02-02-2021$

\section{Resumen}

Introducción: La presente investigación tiene como objetivo construir y determinar las propiedades psicométricas del "Inventario de Respuestas Emocionales Negativas y Perturbadoras en Contextos de Pandemias (IREN-35)" en población adulta mexicana. Dicho instrumento identifica la presencia de emociones negativas relacionadas con la exposición directa o indirecta al virus (SARS-CoV-2), incluida la experiencia de aislamiento físico y social que éste provoca.

Método: Se realizó un estudio exploratorio, descriptivo y transversal con metodología cuantitativa. Con el fin de determinar la validez de constructo, se realizó un análisis factorial exploratorio, así como la identificación de los coeficientes de confiabilidad total y por factores. Para la validación convergente se utilizó un análisis de correlación de Pearson entre el IREN-35 y el Cuestionario General de Salud de Goldberg. El proceso de validación se llevó a cabo durante la pandemia de COVID-19 (coronavirus) en México, en la fase dos de propagación del virus.

Resultados: Los resultados globales indican que el IREN-35 es un instrumento válido y confiable para identificar emociones negativas y presenta un buen desempeño psicométrico en población mexicana adulta. El instrumento está constituido por cuatro factores, con un alfa de Cronbach global de .96: 1) Emociones negativas por "distanciamiento físico" y/o social; 2) Emociones por 
"pérdida" de estabilidad o bienestar; 3) Emociones por percepción de "amenaza"; y 4) Emociones por percepción de "peligro intenso".

Discusión o conclusión: El IREN-35 tiene una utilidad preventiva en el sentido de que puede identificar la presencia de emociones perturbadoras, susceptibles de contribuir -con el transcurrir del tiempo y si su intensidad aumenta- en la formación de algún tipo de malestar emocional (factor 1), o trastorno, ya sea depresivo (factor 2), de ansiedad (factor 3), por traumas o factores de estrés (factor 4) en el contexto de una pandemia.

\section{Abstract}

Introduction: The present research aims to construct and determine the psychometric properties of the "Inventory of Negative and Disturbing Emotional Responses in Pandemic Contexts (IREN35)" in Mexican adult population. This instrument identifies the presence of negative emotions related to direct or indirect exposure to the virus (SARS-CoV-2), including the experience of physical and social isolation that this causes.

Method: An exploratory, descriptive and cross-sectional study was carried out with quantitative methodology. In order to determine construct validity, an exploratory factor analysis was performed, as well as the identification of the total and factor reliability coefficients. For convergent validation, a Pearson correlation analysis was used between IREN-35 and Goldberg's General Health Questionnaire. The validation process was carried out during the COVID-19 (coronavirus) pandemic in Mexico, in phase two of the virus's spread.

Results: The global results indicate that the IREN-35 is a valid and reliable instrument to identify negative emotions, and presents a good psychometric performance in the adult Mexican population. The instrument consists of four factors with a global Cronbach's alpha of .96: 1) Negative emotions due to "physical" and/or social distancing; 2) Emotions due to "loss" of stability or well-being; 3) Emotions due to perceived "threat"; and 4) Emotions due to perception of intense "danger".

Discussion or conclusion: The IREN-35 has a preventive utility in the sense that it can identify the presence of disturbing emotions, likely to contribute -with the passage of time and if their intensity increases- in the formation of some type of emotional distress (factor 1), or depressive disorder (factor 2), anxiety disorder (factor 3), trauma or stress factors (factor 4) in the context of a pandemic. 


\section{Introducción}

Con más de dos millones de muertes por el virus SARS-CoV-2 y 102 millones de personas contagiadas en todo el mundo, los efectos mortíferos del coronavirus siguen creciendo de manera sostenida (Organización Mundial de la Salud, 2021; Real Clear Politics, 2021). En el caso particular de América Latina, la cifra de decesos asciendió a 584 mil, y el número de personas contagiadas superó los 18 millones de casos al 28 de enero de 2021 (Organización Mundial de la Salud, 2021; Real Clear Politics, 2021; Reuters, 2021).

La mortalidad de la COVID-19 ha sido de tal magnitud en todo el mundo, que ya ha superado al número de muertos de algunos desastres naturales más mortíferos de la historia reciente, incluyendo el tsunami de Asia Oriental en 2004 (230 mil muertes) y el terremoto de Haití en 2010, respecto del cual la estimación más alta fue de 316 mil fallecidos (Flores-Morales, 2020a).

En el caso particular de México, el virus ha colocado a esta nación en los primeros lugares en cuanto al número de fallecimientos absolutos de una lista de 185 países. Al 31 de enero de 2021, México ocupaba el tercer lugar en el mundo -después de Estados Unidos y Brasil-, con 158,074 defunciones; y se ubicaba en el décimo quinto lugar por cada millón de habitantes. Asimismo, México llegó a ocupar el primer lugar en el mundo en cuanto a letalidad, con una tasa de 8.50\% (Real Clear Politics, 2021).

A la par de muertes y contagios, la pandemia de coronavirus ha generado también múltiples impactos colaterales, particularmente en la salud mental de la población (Flores-Morales, 2020b). Uno de los factores que ha generado respuestas emocionales diversas durante la pandemia fue justamente la implementación de cuarentenas y distanciamiento físico en todo el mundo -ya fuera por diagnóstico de enfermedad, posible exposición al virus, o por acciones preventivas. Tal confinamiento ha implicado limitaciones drásticas en la libertad de circulación de los ciudadanos, y/o la suspensión de prácticamente todas las actividades (a excepción de aquellas consideradas prioritarias, como atención médica o la que implica producción de alimentos) (Sandín et al., 2020; Williams et al., 2020 ).

De acuerdo con Brooks et al. (2020), la separación de los seres queridos, la pérdida de libertad, la presencia de incertidumbre y aburrimiento, han sido algunos de los elementos generadores de malestar en la población mundial en el contexto de la COVID-19, lo que ha conducido en algunos lugares o en ciertos grupos poblacionales al rechazo sistemático de las medidas de aislamiento. 
Se ha reportado, asimismo, que la promoción del distanciamiento físico y social por la pandemia ha generado desconfianza en ciertos sectores, amplificando estados emocionales negativos (Nicol et al., 2020). Por el contrario, en estudios en los que la población reportó un alto nivel de confianza en médicos que atienden COVID-19 y que mostraron satisfacción con el manejo de la información sobre la pandemia, han presentado niveles estables y poco variables de estrés, ansiedad y depresión a lo largo del tiempo (Wang et al., 2020). Asimismo, cuando percibieron a las políticas de confinamiento como medidas adecuadas que reforzaron la sensación de control, los niveles de estrés disminuyeron considerablemente (Milman et al., 2020).

En el caso particular de México, se ha reportado que durante la pandemia de la COVID-19 aumentaron los síntomas de ansiedad y depresión en el país. En el primer caso, se identificó un incremento de $17 \%$, pasando de $33 \%$ a 50\% en una muestra mexicana. En el caso de los síntomas depresivos, estos aumentaron de 15\% a 28\% (Priego-Parra et al., 2020). Por su parte, GonzálezRamírez et al. (2020) identificaron que el $27.7 \%$ de su muestra presentó síntomas de Estrés Postraumático durante la segunda fase de la COVID-19 en México. Adicionalmente encontraron que 22\% presentó pensamientos intrusivos, 22.3\% síntomas evitativos, y 12.2\% hiperactivación. Algo similar se encontró en otros estudios a nivel mundial, en donde se observó que la pandemia también había tenido un efecto nocivo sobre el bienestar emocional de las personas, la calidad del sueño, y los niveles de ansiedad y desarrollo de síntomas de estrés postraumático (Sandín et al., 2020).

A pesar de las múltiples y variadas respuestas psicológicas generadas por epidemias, desastres naturales o pandemias, los instrumentos de medición utilizados para identificar dichas respuestas han sido con frecuencia los mismos. Dado que estudios realizados en años anteriores encontraron una relación significativa entre el establecimiento de cuarentenas como medidas sanitarias y la presencia de síntomas de estrés postraumático (Brooks et al., 2020), no sorprende, entonces, el uso reiterado y sistemático de escalas de tamizaje para identificar síntomas de distrés, estrés agudo e indicadores de Estrés Postraumático (PTSD), en contextos de pandemias y desastres.

Entre las escalas más utilizadas en dichos contextos se encuentran el "Impact of Event Scale Revised (IES-R)” (El-Zoghby et al., 2020; González-Ramírez et al., 2020; Hao et al., 2020; Parrado-González \& León-Jariego, 2020; Samaniego et al., 2020; Tan et al., 2020; Varshney et al., 2020; Wang et al., 2020) y el "Post-traumatic Stress Disorder Checklist-Reduced version (PCL-C2)" para evaluar PTSD (González-Sanguino et al., 2020); y el "Kessler Psychological Distress 
Scale (K10)" para evaluar distrés (Moccia et al., 2020). Dichas escalas, a su vez, han sido ampliamente utilizadas ante otros eventos traumáticos como desastres naturales y terremotos (Nagamine et al., 2018; Stratta et al., 2013), inundaciones (Bei et al., 2013) y huracanes (LaJoie et al., 2010).

Hasta el momento, entre los instrumentos más utilizados en investigaciones sobre el impacto psicológico de la COVID-19, se ubican las escalas para evaluar sintomatología ansiosa, como el "Generalized Anxiety Disorder Scale" (GAD en sus diferentes versiones) (GonzálezSanguino et al., 2020; Samaniego et al., 2020), sintomatología depresiva con el "Patient Health Questionnaire (PHQ 9 o 2)" (González-Sanguino et al., 2020; Samaniego et al., 2020); o ambas, con la escala "Depression, Anxiety and Stress Scales (DASS-21)" (Hao et al., 2020; Orellana \& Orellana, 2020; Tan et al., 2020; Wang et al., 2020). En otras investigaciones se ha empleado el "Cuestionario General de Salud de Goldberg (GHQ-12)”, con la intención de evaluar de forma más integral el bienestar general y la presencia de ciertos estados emocionales asociados a malestares psicológicos debido a la pandemia (Parrado-González \& León-Jariego, 2020).

Aunque han sido numerosos los instrumentos psicométricos utilizados en el contexto de la pandemia de COVID-19, la mayoría se ha enfocado en la identificación de síntomas y, en consecuencia, ha subestimado el estudio de respuestas "normales", como podrían ser el vasto conglomerado de emociones que presenta la población ante un evento de esta naturaleza. En este sentido, el estudio de las emociones cobra especial relevancia en contextos pandémicos, debido a que éstas representan señales que desencadenan un conjunto de respuestas experienciales y de comportamiento, tanto a nivel del sistema fisiológico central como periférico. Dichas respuestas, en efecto, se desencadenan ante un evento externo o interno que le indican al individuo que algo importante podría estar en juego (Rottenberg \& Gross, 2007).

Y es que, en efecto, desde que William James (1890) categorizó a las emociones como "respuestas corporales" o como "reacciones fisiológicas", pasando por los múltiples modelos que han dado cuenta de ellas a lo largo de la historia reciente -por ejemplo, las teorías de la sensación, las conductuales, evolutivas, cognoscitivas, constructivistas, e incluso las socioculturales- el universo de las emociones se ha ubicado como una de las áreas de estudio más importantes de la psicología y de otras disciplinas sociales (Calhoun \& Solomon, 1989; Le Breton, 2013; Ortony, et al., 1996; Marina, 1996). En este sentido, como lo señala Chóliz (2005), la emoción es una experiencia multidimensional con al menos tres vertientes básicas: a) fisiológico/adaptativo, b) 
cognitivo/subjetivo; y c) conductual/expresivo. David Le Breton (2013) añadiría una cuarta: la constructivo-sociocultural.

Desde estas perspectivas, las respuestas emocionales pueden ser abordadas como reacciones adaptativas a un amplio espectro de condicionantes y estímulos ambientales -sin por ello olvidar que también son producto de construcciones socioculturales-, las cuales no tendrían por qué categorizarse necesariamente como psicopatológicas (en particular las denominadas "emociones negativas").

Sin embargo, cuando el cúmulo de emociones perturbadoras se prolonga en el tiempo, generando malestar persistente, lo que en un inicio representaba un proceso adaptativo y regulatorio, suele transformarse en un elemento desestabilizador del self, cuyo desenlace puede producir malestar psicológico en las personas. En este sentido, las pandemias -en su condición de estímulos evocadores- son capaces de activar señales emocionales de alerta en el organismo las cuales, si bien a corto plazo representan respuestas normales y adaptativas, a largo plazo podrían ser dañinas para los individuos que las experimentan si estas son permanentes en términos temporales e intensas en su magnitud.

Entre las muchas emociones que pueden detonar las pandemias, el miedo individual y colectivo ante el riesgo latente de un eventual contagio es el que más figura en las investigaciones (Sandín et al., 2020; Shalev \& Shapiro, 2020). En consonancia con esto, Sandín et al. (2020), crearon la Escala de Miedos al Coronavirus $($ EMC) (coeficiente de fiabilidad alfa $(\alpha)=.89$ ) de 18 ítems para evaluar los principales miedos en población española. Los autores identificaron miedos a la propia enfermedad, a la carencia, al aislamiento, y al aspecto laboral y económico. Asimismo, dichos autores utilizaron la Escala PANAS de Afecto Positivo y Negativo de 20 ítems ( $\alpha=.91 ; \alpha=$ .90), y revelaron puntuaciones importantes de afecto negativo en su población, resultados igualmente reportados por Somma et al., (2020), con el uso de la "Strengths and Difficulties Questionnaire Emotional Problems Scale (SDQ EPS)".

No obstante, la creación de una escala como el IREN-35 que logre identificar "emociones perturbadoras" (que no se limiten al miedo y al estrés), y que al mismo tiempo se ajuste a la población mexicana adulta y a lo que ésta experimenta en contextos de desastres y pandemias particularmente de COVID-19- resulta de gran relevancia social y clínica.

En este sentido, en la presente investigación entendemos por "respuestas emocionales perturbadoras" aquellas experiencias subjetivas que modifican o alteran el orden o la quietud de 
una persona. La "perturbación" describe cierto periodo de inestabilidad que vulnera un estado que se considera como óptimo o normal. Por lo general la perturbación se produce ante una situación traumática o que pone en riesgo la integridad de los individuos, la cual regularmente cambia o modifica su estado de ánimo. La palabra perturbación proviene del latín perturbātiō, y remite a cierta alteración, confusión o conmoción que rompe con un estado previamente estable (Lewis \& Short, 1879). Por ello, la perturbación (disturbing en inglés) no tiene necesariamente un carácter psicopatológico.

En suma, el impacto emocional que ha experimentado la población mundial ante la pandemia de COVID-19, así como la vivencia de aislamiento físico y social que provoca, han sido poco estudiados en México y América Latina. Por ello, el objetivo del presente estudio es identificar las principales emociones perturbadoras que ha experimentado la población adulta mexicana en el contexto de la COVID-19, a través de la construcción y aplicación del "Inventario de Respuestas Emocionales Negativas y Perturbadoras en Contextos de Pandemias (IREN-35)"; así como determinar las propiedades psicométricas de dicha escala.

\section{Método}

\section{Diseño}

El presente estudio forma parte de una investigación más amplia sobre las respuestas emocionales que ha experimentado la población mexicana adulta en contextos de pandemias y desastres naturales. Para esta primera etapa de validación de instrumentos, se realizó un estudio exploratorio, descriptivo y transversal con metodología cuantitativa (Clark-Carter, 2002), en donde se analizó la validez de constructo y la validez convergente del "Inventario de Respuestas Emocionales Negativas y Perturbadoras en Contextos de Pandemias (IREN-35)", así como su coeficiente de confiabilidad global y por factores. De acuerdo con los criterios de Kerlinger y Lee (2002), la presente investigación es de tipo metodológico en tanto que evalúa la validez y confiabilidad de pruebas psicométricas.

\section{Participantes}

Se empleó una muestra intencional por invitación en cadena por redes (Hernández, et al., 2014). Participaron 502 personas adultas de la Zona Metropolitana de la Ciudad de México (ZMCM), cuyas edades oscilaron entre 18 y 75 años $(\overline{\mathrm{x}}=32) .66 .3 \%$ fueron adultos jóvenes (entre 18 y 35 
años), $31.3 \%$ adultos maduros (entre 36 y 64 ), y únicamente $2.4 \%$ de los participantes fueron adultos mayores, con una edad de 65 años o más.

$72.2 \%$ de los participantes fueron mujeres y $27.3 \%$ hombres. El estado civil se distribuyó de la siguiente forma: $69.3 \%$ solteros, $26.1 \%$ casados o en unión libre, $3.4 \%$ divorciados o separados y $1.2 \%$ viudos. En cuanto a la escolaridad, 0.4\% únicamente terminó la educación primaria, $1.8 \%$ cursó secundaria, $12.5 \%$ preparatoria o equivalente, $44 \%$ estaba cursando la licenciatura (es decir, eran estudiantes universitarios), 31.1\% terminó una licenciatura y 10.2\% cursó o estaba cursando un posgrado al momento del levantamiento de los datos.

En un inicio se invitó a un total de 122 estudiantes universitarios de dos universidades de la Ciudad de México (una pública y otra privada) para que participaran en el estudio. Una vez que los alumnos aceptaron la invitación y respondieron los instrumentos, se les solicitó que, si no tenían inconveniente, compartieran el formulario con sus familiares y amigos cercanos con el fin de ampliar el espectro del estudio. De esta forma, se pudo alcanzar una muestra final de 502 participantes de la Zona Metropolitana de la Ciudad de México (ZMCM), que fue la región geográfica más afectada del país por la COVID-19 (Secretaría de Salud, 2020).

Los criterios de inclusión para este estudio fueron los siguientes: 1) Tener 18 años o más; 2) Vivir en la Zona Metropolitana de la Ciudad de México (ZMCM) al momento de la pandemia (puesto que esta fue la región geográfica más afectada del país); 3) Estar respetando el distanciamiento físico sugerido por las autoridades sanitarias en el contexto de la COVID-19. Los criterios de exclusión fueron los siguientes: 1) Ser menor de edad; y 2) Vivir en cualquier otro estado de la república que no fuera la ZMCM.

\section{Instrumentos}

Se aplicó el "Inventario de Respuestas Emocionales Negativas y Perturbadoras en Contextos de Pandemias (IREN)", el cual se desarrolló a partir de un proceso de tres etapas.

En la primera etapa, un grupo de seis doctores en psicología y expertos en el tema de las emociones, sentimientos y afectos, se reunió para desarrollar una lista preliminar de las principales emociones que, a su juicio profesional, podrían experimentar las personas al estar vivido de manera directa una pandemia. Como resultado de este primer ejercicio, el equipo identificó 249 emociones preliminares susceptibles de estar presentes en una crisis pandémica. 
En una segunda etapa, llevada a cabo una semana después, cuatro expertos (dos especialistas del equipo inicial ya no pudieron participar en esta segunda fase) realizaron un nuevo análisis con el objetivo de filtrar y reducir la primera lista a un número significativamente menor, dejando únicamente las que, a su juicio clínico y profesional, podrían ser las emociones más recurrentes. En esta segunda etapa, los especialistas comprimieron la lista a 90 emociones. La versión de 90 emociones del instrumento fue la que se aplicó a los 502 participantes voluntarios del presente estudio - la cual constituyó la tercera etapa de esta investigación-, y a partir de ella se realizaron los análisis estadísticos que condujeron a la versión final del IREN con 35 reactivos.

En el instrumento, al participante se le informó que se le presentaría una lista de emociones y sentimientos que podrían estar experimentando en el contexto de la pandemia de coronavirus (COVID-19). Se le solicitó que por favor respondiera qué tan presente había estado cada una de estas emociones en su vida diaria durante las últimas dos semanas. El inventario se respondió usando una escala Likert de cinco opciones: "Nada presente" (1), "Poco presente" (2), "Más o menos presente" (3), "Muy presente" (4) y "Demasiado presente" (5).

Por otra parte, con el fin de llevar a cabo la validación convergente/divergente, durante la tercera etapa de esta investigación también se aplicó la versión mexicana del Cuestionario General de Salud de Goldberg y Williams de 12 reactivos (CGS) (Solís-Cámara et al., 2016). El CGS evalúa la salud autopercibida, el estado de bienestar general, y particularmente la presencia de ciertos estados emocionales asociados a malestares psicológicos. La versión estandarizada para población mexicana está constituida por una dimensión (bienestar/malestar psicológico) y tiene un alfa total de .81 .

Adicionalmente a los dos instrumentos arriba descritos, en la tercera etapa también se aplicó un cuestionario sociodemográfico, en donde se les preguntó su edad, género, estado civil, lugar de residencia, escolaridad y si estaban o no acatando el distanciamiento social/físico sugerido por las autoridades de salud del país. Esta última pregunta se planteó a través de una opción dicotómica: sí/no.

\section{Procedimiento}

La aplicación de instrumentos se realizó a través de la Plataforma de Formularios de Google debido

a las restricciones sanitarias que impedían el contacto físico entre las personas. Para el proceso de validación del IREN de la tercera etapa, el levantamiento de datos se realizó del 25 de marzo al 20 
de abril de 2020, en el contexto de la Fase 2 de la pandemia por COVID-19 en México. Durante esta fase -identificada como de "transmisión comunitaria"- las autoridades sanitarias del país efectuaron una serie de medidas mitigadoras más estrictas para evitar la propagación del virus, como la suspensión de eventos masivos y concentraciones de personas en espacios reducidos (incluidas todas las escuelas, institutos y universidades del país), así como optar por el aislamiento voluntario.

La Fase 2 de la pandemia fue implementada a nivel nacional el 23 de marzo, justamente dos días antes de que se comenzara con el levantamiento de datos del presente estudio (Gobierno de México, 2020). Pese a que en términos oficiales el aislamiento físico y social inició en la tercera semana de marzo, en ciertos lugares de la Ciudad de México el distanciamiento voluntario de la población comenzó tres semanas antes -el 28 de febrero de 2020-justamente cuando se presentó el primer caso de COVID-19 en México. A partir de esa fecha, el gobierno federal intensificó campañas que ya promovían el distanciamiento físico de las personas.

\section{Análisis estadístico}

Los datos obtenidos se analizaron en el programa SPSS versión 25 y se emplearon diferentes procedimientos estadísticos. Para verificar la validez psicométrica de la escala, se llevaron a cabo las propuestas de Reyes-Lagunes y García-Barragán (2008).

En primera instancia se realizó un análisis de frecuencias y de discriminación de reactivos por el método de grupos contrastados ( $t$ de Student para muestras independientes). Para iniciar el proceso de validez de la escala, se empleó el índice de adecuación de la muestra de KMO (KaiserMeyer-Olkin) y la prueba de esfericidad de Bartlett. Posteriormente, se realizó un análisis factorial exploratorio con rotación ortogonal varimax, bajo el método de extracción de máxima verosimilitud. Por último, se examinó la confiabilidad total y por factores del IREN mediante el índice Alfa de Cronbach.

Para evaluar la validez convergente/divergente del IREN, se identificaron los coeficientes de correlación de Pearson entre los puntajes totales y las dimensiones de los dos instrumentos aplicados, utilizando los criterios propuestos por Cohen (1992). 


\section{Consideraciones éticas}

En el formulario que se les envió a los participantes a través de la Plataforma de Google, se les explicó el objetivo del estudio y la importancia que representaba para los autores su participación. Se decidió utilizar esta modalidad de levantamiento de datos a través de internet, debido a que todo el país se encontraba en distanciamiento físico, por lo que resultaba imposible realizar las aplicaciones de los instrumentos cara a cara. A los participantes se les garantizó estricta confidencialidad, añadiendo que, si en algún momento de la aplicación de los instrumentos decidían retirarse, estaban en su completo derecho de hacerlo. Una vez aprobado su consentimiento informado, se procedió con el proceso de recolección de datos.

\section{$\underline{\text { Resultados }}$}

Como primer paso y con el objetivo de obtener la validez de constructo del IREN, se identificaron las frecuencias de cada ítem y se llevó a cabo un análisis de discriminación de reactivos por el método de grupos contrastados, utilizando la prueba t de Student para muestras independientes. Como resultado del análisis, se encontró que 12 de los 90 reactivos originales no discriminaron, de manera que fueron eliminados, quedando 78 emociones. Las emociones que no discriminaron y que no obtuvieron un p valor menor a 0.05 fueron las siguientes: alegría, alivio, armonía, confianza, control, diversión, entusiasmo, felicidad, paciencia, paz, seguridad y serenidad.

Posteriormente, se procedió a realizar un análisis factorial exploratorio, utilizando el método de extracción de máxima verosimilitud con rotación ortogonal varimax. Se obtuvo un coeficiente KMO (Kaiser-Meyer-Olkin) de .96, ubicándose muy por encima del .50 recomendado (Kaiser, 1970). Los resultados de la prueba de esfericidad de Bartlett también fueron significativos $(\mathrm{p}=.00)$.

Al realizar el análisis factorial, se obtuvo una estructura final de cuatro dimensiones, compuesta en su conjunto por 35 de las 78 emociones enlistadas, explicando el $56.49 \%$ de la varianza, con indicador de bondad de ajuste Chi cuadrada $=1052.931, \mathrm{gl}=461, \mathrm{p}=.00$. Los 35 reactivos definitivos obtuvieron cargas mayores a .40 y ninguno se ubicó en más de una dimensión (Tabla 1). 
Tabla 1. Análisis factorial exploratorio del IREN-35.

Table 1. Exploratory factor análisis.

\begin{tabular}{|c|c|c|c|c|}
\hline & $\begin{array}{l}\text { Emociones negativas } \\
\text { por distanciamiento } \\
\text { físico y/o social }\end{array}$ & $\begin{array}{c}\text { Emociones } \\
\text { negativas por } \\
\text { pérdida/ausencia } \\
\text { de una condición } \\
\text { de estabilidad o } \\
\text { bienestar }\end{array}$ & $\begin{array}{l}\text { Emociones } \\
\text { negativas por } \\
\text { percepción de } \\
\text { amenaza }\end{array}$ & $\begin{array}{l}\text { Emociones } \\
\text { negativas } \\
\text { por } \\
\text { percepción } \\
\text { de peligro } \\
\text { intenso }\end{array}$ \\
\hline Varianza explicada & $18.81 \%$ & $17.97 \%$ & $11.67 \%$ & $8.03 \%$ \\
\hline Alfa de Cronbach & .935 & .910 & .886 & .908 \\
\hline Media & 2.12 & 1.67 & 2.52 & 1.5 \\
\hline Desviación Estándar & 0.82 & 0.70 & 0.90 & 0.87 \\
\hline Fastidio & 0.746 & 0.251 & 0.190 & 0.103 \\
\hline Aburrimiento & 0.722 & 0.016 & -0.008 & 0.097 \\
\hline Desgano & 0.715 & 0.365 & 0.180 & 0.074 \\
\hline Desmotivación & 0.709 & 0.386 & 0.179 & 0.132 \\
\hline Pereza & 0.686 & 0.037 & 0.244 & 0.000 \\
\hline Impaciencia & 0.614 & 0.178 & 0.374 & 0.205 \\
\hline Irritabilidad & 0.608 & 0.241 & 0.351 & 0.129 \\
\hline Amargura & 0.586 & 0.360 & 0.169 & 0.085 \\
\hline Desesperación & 0.577 & 0.378 & 0.256 & 0.228 \\
\hline Soledad & 0.564 & 0.208 & 0.171 & 0.115 \\
\hline Insatisfacción & 0.523 & 0.375 & 0.334 & 0.064 \\
\hline Tristeza & 0.510 & 0.381 & 0.381 & 0.194 \\
\hline Pesimismo & 0.481 & 0.398 & 0.377 & 0.242 \\
\hline Hastío & 0.468 & 0.356 & 0.206 & 0.043 \\
\hline Apatía & 0.448 & 0.056 & 0.004 & 0.006 \\
\hline Desconsuelo & 0.198 & 0.781 & 0.211 & 0.184 \\
\hline Desdicha & 0.289 & 0.742 & 0.097 & 0.187 \\
\hline Desamparo & 0.157 & 0.708 & 0.241 & 0.128 \\
\hline Desaliento & 0.392 & 0.676 & 0.241 & 0.102 \\
\hline Desasosiego & 0.284 & 0.637 & 0.278 & 0.103 \\
\hline Desconfianza & 0.106 & 0.610 & 0.352 & 0.218 \\
\hline Desesperanza & 0.324 & 0.600 & 0.279 & 0.239 \\
\hline Descontrol & 0.273 & 0.553 & 0.243 & 0.174 \\
\hline Recelo & 0.138 & 0.496 & 0.270 & 0.139 \\
\hline Duelo & 0.135 & 0.463 & 0.201 & 0.194 \\
\hline Incertidumbre & 0.287 & 0.272 & 0.642 & 0.166 \\
\hline Preocupación & 0.168 & 0.275 & 0.600 & 0.362 \\
\hline Inquietud & 0.349 & 0.277 & 0.594 & 0.185 \\
\hline Vulnerabilidad & 0.143 & 0.331 & 0.590 & 0.124 \\
\hline Inseguridad & 0.178 & 0.366 & 0.582 & 0.245 \\
\hline Impotencia & 0.276 & 0.340 & 0.532 & 0.205 \\
\hline Fragilidad & 0.277 & 0.383 & 0.434 & 0.216 \\
\hline Pavor & 0.108 & 0.228 & 0.204 & $\mathbf{0 . 8 3 5}$ \\
\hline Pánico & 0.151 & 0.244 & 0.316 & 0.793 \\
\hline Terror & 0.123 & 0.312 & 0.225 & 0.745 \\
\hline
\end{tabular}

Nota: Se utilizó el método de extracción de máxima verosimilitud, con rotación Varimax. La rotación convergió en seis iteraciones.

Note: The maximum likelihood extract method was used, with Varimax rotation. The rotation converged in six iterations. 
Al primer factor se le denominó "Respuestas emocionales por distanciamiento físico y/o social", ya que se refieren al conjunto de emociones/sensaciones relacionadas o vinculadas con el aislamiento físico y con la imposibilidad de salir de casa debido al riesgo de un eventual contagio. Las emociones con cargas factoriales más elevadas de esta dimensión fueron el fastidio (.746), aburrimiento (.722) y desgano (.715). Las de cargas medias, irritabilidad (.608), desesperación (.577) y soledad (.564), mientras que las cargas más bajas correspondieron a la tristeza (.510), pesimismo (.481) y hastío (.468).

El segundo factor fue identificado como "Respuestas emocionales por pérdida o ausencia de una condición de estabilidad/bienestar preexistente". Esta dimensión alude principalmente a la pérdida de una sensación de estabilidad, posiblemente presente antes de la pandemia, y que desapareció como consecuencia de ella. Las emociones sobresalientes de este factor fueron el desconsuelo (.781), el desamparo (708), la desesperanza (.600), el descontrol (.553), el recelo (.496) y el duelo (.463).

El tercer factor alude a un conjunto de "Emociones negativas por percepción de amenaza". Las principales emociones de este factor fueron la incertidumbre (.642), preocupación (.600), inseguridad (.582), sensación de vulnerabilidad (.590), impotencia (.532) y fragilidad (.434).

Finalmente, el cuarto factor fue denominado "Emociones negativas por percepción de peligro intenso". Este último factor se refiere a la percepción de un riesgo considerable a la integridad y salud de las personas, el cual puede colocar en peligro su propia vida. Las emociones que configuran esta dimensión es el pavor (.835), el pánico (.793) y el terror (.745).

A los dos últimos factores los separa únicamente una percepción de la "intensidad" del riesgo; mientras el tercero es más "leve" y alude a una "amenaza" o posible afectación, el cuarto remite a la percepción de un "peligro intenso" derivado de la pandemia.

En cuanto a la confiabilidad del inventario, el Alfa de Cronbach total fue .96. El coeficiente del primer factor fue .93 , del segundo .91 , del tercero .88 , y del cuarto .90 .

También se identificaron correlaciones significativas entre los cuatro factores con un $\mathrm{p}<.01$ (Tabla 2). La correlación más baja se estableció entre las "Respuestas emocionales por distanciamiento físico y/o social" y las "Emociones negativas por percepción de peligro intenso" $(\mathrm{r}=440)$, mientras que la más alta se reportó entre las "Respuestas emocionales por pérdida o ausencia de una condición de estabilidad/bienestar preexistente" y las "Emociones negativas por percepción de amenaza" ( $\mathrm{r}=.734)$. (Tabla 2). 
Tabla 2. Correlaciones entre los factores del IREN-35.

Table 2. Correlations between IREN-35 factors.

\begin{tabular}{|c|c|c|c|c|c|}
\hline & Factor & 1 & 2 & 3 & 4 \\
\hline 1 & $\begin{array}{l}\text { Emociones por } \\
\text { distanciamiento } \\
\text { físico/social. }\end{array}$ & -- & .690 & .682 & .440 \\
\hline 2 & $\begin{array}{l}\text { Emociones de pérdida o } \\
\text { ausencia de una condición } \\
\text { de estabilidad o bienestar. }\end{array}$ & & -- & .734 & .553 \\
\hline 3 & $\begin{array}{l}\text { Emociones por percepción } \\
\text { de amenaza. }\end{array}$ & & & -- & .600 \\
\hline 4 & $\begin{array}{l}\text { Emociones por percepción } \\
\text { de peligro intenso. }\end{array}$ & & & & -- \\
\hline
\end{tabular}

Las correlaciones intermedias se establecieron entre las "Respuestas emocionales por distanciamiento físico y/o social" y las "Respuestas emocionales por pérdida o ausencia de una condición de estabilidad/bienestar preexistente" ( $\mathrm{r}=.690)$. Y entre este último factor y las "Emociones negativas por percepción de peligro intenso" ( $\mathrm{r}=.553)$.

También se encontró una correlación alta entre las "Respuestas emocionales por distanciamiento físico y/o social" y las "Emociones negativas por percepción de amenaza" $(\mathrm{r}=.682)$. Y, finalmente, se identificó una correlación entre este último factor y las "Emociones negativas por percepción de peligro intenso" ( $\mathrm{r}=.600)$.

Con respecto a la validación convergente, se identificó una correlación alta y significativa entre el IREN-35 y el Cuestionario General de Salud de Goldberg ( $r=.782$ ). En cuanto a sus dimensiones, los cuatro factores correlacionaron de manera positiva con el CGS. El primer factor presentó una correlación alta $(\mathrm{r}=.770)$; el segundo factor tuvo un puntaje ligeramente inferior pero igualmente alto $(\mathrm{r}=.674)$; mientras que el tercer y cuarto factores se ubicaron con un puntaje de .615 y .444 respectivamente. El coeficiente de confiabilidad del Cuestionario General de Salud identificado en este estudio fue .88 .

\section{Discusión o conclusiones}

El presente estudio tuvo como objetivo construir y validar un inventario que identifique la presencia de emociones negativas o perturbadoras relacionadas, vinculadas, construidas y/o desencadenadas por la exposición directa o indirecta a una pandemia como la de COVID-19, en un contexto mexicano. 
Los resultados globales indican que el IREN-35 es un instrumento válido y confiable. Cada uno de los factores y reactivos que lo constituyen cumplieron con las propiedades psicométricas necesarias (cargas factoriales por encima de .40, alfas de Cronbach iguales o mayores a .70, correlaciones significativas, etc.). La confiabilidad total reportada fue de .96 , lo cual demuestra que es un instrumento robusto y confiable para población mexicana adulta. Los coeficientes reportados en cada una de las dimensiones también fueron elevados $(.88, .90, .91$ y .93$)$, por lo que se ubicaron muy por encima del recomendado por Nunnally y Berstein (1994), que es de .70.

La validez convergente también confirmó la robustez del Inventario, al identificar correlaciones positivas, altas y significativas con el Cuestionario General de Salud de Goldberg $(\mathrm{r}=.782)$. El CGS también correlacionó por separado con las cuatro dimensiones del IREN-35.

En este sentido, el inventario puede tener una utilidad preventiva en la configuración de determinados trastornos, ya que al identificarse con cierta anticipación la presencia de emociones negativas y perturbadoras en un contexto pandémico, resultaría posible articular estrategias de intervención más adecuadas y eficientes en el cuidado de la salud mental de las personas. Como se sabe, si las emociones negativas y perturbadoras son intensas en su manifestación y persistentes en el tiempo, entonces éstas podrían coadyuvar en la configuración de algún tipo de trastorno, particularmente de ansiedad, depresivo o traumático.

El primer factor identificado en esta investigación -emociones por "distanciamiento físico/social" - cobra sentido y se configura en el contexto de una cuarentena voluntaria, debido a los riesgos de contagio que generó la pandemia. La intensidad y rapidez en la propagación del virus, en efecto, obligó a la población de muchas partes del mundo a refugiarse en sus hogares, y el alargamiento de la cuarentena -con criterios cada vez más estrictos de distanciamiento socialllegó a extenderse en ciertos países por más de cuatro meses consecutivos.

El confinamiento -ya fuera voluntario u obligatorio, dependiendo de las medidas adoptadas por cada país- generó condiciones psicosociales que coadyvaron en la emergencia de emociones negativas perturbadoras, las cuales trastocaron la vida cotidiana de muchos ciudadanos. La imposibilidad de salir de casa, las interacciones familiares que se generaron en los hogares, las fricciones entre sus miembros, el estrés por realizar trabajo en casa (home office), los cuidados de niños y niñas de manera simultánea a las labores de sustento, la emergencia de nuevas rutinas, y en ocasiones la irrupción de conductas violentas al interior de los hogares, han sido algunos de los 
condicionantes en el surgimiento de este grupo de emociones negativas perturbadoras (FloresMorales, 2020b).

En un contexto cultural como el de México, en donde la población está habituada a la interacción física y afectiva con el prójimo, la imposibilidad de salir de casa representó un duro golpe a su cotidianidad. La dificultad para convivir con amigos y familiares externos por el riesgo latente a la transmisión, les exigió adaptarse a nuevas formas y dinámicas de relación e integración -regularmente de tipo virtual-, las cuales no siempre se asimilaron de forma adaptativa (PriegoParra et al., 2020).

En este sentido, las emociones perturbadoras más recurrentes vinculadas con el "distanciamiento físico y social" propio de este primer factor, fueron la tristeza, desmotivación, aburrimiento, soledad, desesperación, fastidio e irritabilidad. Todas estas emociones se agruparon en una primera dimensión tras el análisis factorial exploratorio y pueden estar asociadas a un eventual malestar psicoemocional de acuerdo con los criterios de Goldberg (Solís-Cámara et al., 2016)

En particular, la irritabilidad y la soledad - presentes en este primer factor emergente-, son capaces de generar consecuencias altamente nocivas en la salud física y emocional de las personas. Por ejemplo, las fricciones cotidianas al interior de los hogares -derivadas justamente de la interacción diaria e intermitente de sus integrantes- pueden generar irritación en algunos de ellos $\mathrm{y}$, en consecuencia, el surgimiento de agresiones y violencia intrafamiliar (particularmente de género).

De acuerdo con la Organización de las Naciones Unidas (ONU), las medidas de aislamiento llevadas a cabo durante la pandemia han tenido repercusiones diversas, como altos niveles de estrés, desempleo e inseguridad económica. No obstante, también aumentaron paralelamente los niveles de violencia contra mujeres, niñas y niños en el ámbito doméstico (ONU, 2020).

En el caso particular de México, un estudio del Centro de Investigación y Docencia Económica (CIDE, 2020) encontró que, por ejemplo, durante el mes de marzo de 2017 y 2018, el gobierno de la Ciudad de México recibió 201 y 279 llamadas telefónicas respectivamente denunciando violencia de género. Estas cifras se triplicaron en el mismo mes durante 2020, llegando a 811 denuncias telefónicas. A nivel nacional, las denuncias de violencia contra las mujeres pasaron de 19 mil 183 en enero, a 21 mil 727 en febrero, y 26 mil 171 en marzo de 2020, justo en el marco del distanciamento por el coronavirus (Martínez, 2020). 
Con respecto a la presencia de la soledad -justamente el polo opuesto de la interacción física-, un estudio de la Escuela Bloomberg de Salud Pública y de la Universidad Johns Hopkins de los Estados Unidos, concluyó que en dicha investigación los encuestados presentaron mayores indicadores de soledad durante la pandemia de COVID-19 que durante el año 2018, asociados justamente al aislamiento social (McGinty et al., 2020). Por su parte, Luchetti et al. (2020) encontraron en una muestra nacional en Estados Unidos que los adultos mayores experimentaron menos soledad al inicio de la misma, en comparación con los grupos más jóvenes, pero tuvieron un aumento durante la fase aguda del brote.

Es probable que la pandemia de COVID-19 esté generado sentimientos de soledad en algunas personas al disminuir drásticamente sus interacciones rutinarias hacia el exterior, y al sustituir el contacto físico con otros modos de comunicación virtuales que podrían estar aumentado, paradójicamente, su intensidad (Luchetti et al., 2020; National Academies of Sciences, Engineering, and Medicine, 2020). Como lo señalan Trad et al., (2020), la soledad es la percepción subjetiva de un déficit en la conexión social. Por el contrario, el aislamiento social, es una medida objetiva basada en el tamaño de la red social o la frecuencia de las interacciones sociales. Si no se controla, la soledad puede transformarse en un potente factor de riesgo para la salud física y mental de las personas (Beutel et al., 2017).

Por ejemplo, en un metanálisis de 70 estudios, se encontró que las personas solitarias tenían $26 \%$ más de probabilidad de muerte no suicida, durante un seguimiento medio de siete años (HoltLunstad et al., 2015). Por otra parte, estudios transversales han relacionado a la soledad con tasas más altas de ansiedad, depresión e ideación suicida (Beutel et al., 2017). De acuerdo con HoltLunstad et al. (2015), la soledad prolongada es un factor determinante en la salud -aunque muchas veces es subestimado- ya que coloca a las personas en mayor riesgo de muerte prematura que la propia obesidad, la actividad física inadecuada o la contaminación del aire.

El caso de los adultos mayores es particularmente importante, puesto que ese sector ha sido ubicado como un grupo especialmente vulnerable en el contexto pandémico. Aunque el distanciamiento social es fundamental para mitigar la transmisión de COVID-19, el riesgo que representa la soledad en éste y todos los grupos sociales, debe tomarse en consideración por las múltiples consecuencias negativas que genera (Ward et al., 2020).

El segundo factor identificado en la presente investigación alude a emociones perturbadoras que podrían derivarse de la "pérdida" o "ausencia" de una condición de "bienestar" o "estabilidad" 
preexistente justo antes de la pandemia. Las emociones de desconsuelo, desdicha, desamparo, desaliento, desasosiego, descontrol, desesperanza y duelo remiten, en efecto, a la pérdida de un estado preexistente o a la ausencia de cierta estabilidad arrebatada por la propia pandemia.

El prefijo "des" que articula a la mayoría de estas emociones, refiere justamente a la “inversión” de un periodo de estabilidad o bienestar. De acuerdo con el Diccionario de la Real Academia de la Lengua Española (García, 2007), el prefijo "des-“ denota una "negación” o "inversión" del significado de la palabra simple a la que va antepuesto. En este sentido, el conjunto de emociones negativas propias de esta segunda dimensión alude a la ausencia, "inversión" o negación de "algo" (de un estado, una sensación o una experiencia), que estaba presente poco antes de la pandemia y que terminó por diluirse o desaparecer debido a ella.

Des-consuelo, des-dicha, des-amparo, des-aliento y desa-sosiego remiten, en efecto, a la ausencia de consuelo, dicha, amparo, aliento y sosiego. Es decir, a la negación o pérdida (duelo) de un periodo de estabilidad o bienestar preexistente, arrebatado justamente por la emergencia de la pandemia.

Dichas emociones suelen ser estados anímicos negativos de carácter regularmente depresivo, en donde el elemento preponderante es la pérdida de cierta estabilidad preexistente a la pandemia. En este sentido, el duelo agrupa al conjunto de experiencias de "pérdida" o ausencia de una sensación de control, así como la identificación de cierto grado de vulnerabilidad. En consecuencia, frente a la realidad del contagio y de la posibilidad de morir (en las pandemias todos somos vulnerables), puede surgir la sensación de que no hay consuelo, no hay esperanza, no hay dicha, no hay amparo y no hay control frente a las nuevas condiciones de la pandemia.

En este sentido, un estudio cualitativo enfocado en explorar justamente las percepciones y experiencias de la población general en Reino Unido sobre el distanciamiento social y las medidas de aislamiento relacionadas con la pandemia de COVID-19, tuvo hallazgos similares al de la investigación que aquí se presenta. Los participantes reportaron tener una experiencia de "pérdida" en tres niveles: 1) Sociales y económicos; 2) Interacción social; y 3) Ingresos, estructura y rutina. Esto a su vez condujo a tres pérdidas a nivel psicológico y emocional: 1) Motivación; 2) Significado; y 3) Autoestima. Uno de los participantes del estudio inclusive comparó su experiencia con un proceso de "duelo", pese a que ningún pariente suyo o ser querido había fallecido durante la pandemia (Williams et al., 2020). 
Y es que, en efecto, el COVID-19 ha generado una multiplicidad de cambios y pérdidas en las personas (como se sabe, no necesariamente el fallecimiento de algún ser querido o conocido es la única forma de "pérdida"). De la Hoz (2020) de la Organización Panamericana de la Salud (OPS), por ejemplo, identificó pérdidas en distintos rubros o áreas en el contexto del coronavirus: empleo, vivienda (en caso de tener que mudarse por el riesgo de contagio), libertad, contacto físico con familiares y amigos, salud, seres queridos, estabilidad económica y laboral, espacios de ocio y distracción.

De acuerdo con lo hallado en nuestra investigación, las pérdidas arriba citadas podrían estar promoviendo la configuración de emociones como desconsuelo, desdicha, desamparo, desaliento, desasosiego, desesperanza y descontrol. Si su presencia persiste con el paso del tiempo, algunas de estas respuestas emocionales podrían representar la antesala de algún tipo de trastorno, particularmente el depresivo (American Psychiatric Association, 2013a).

Como se sabe, el trastorno depresivo mayor está caracterizado por la presencia de un fuerte sentimiento de tristeza y una "pérdida" de interés en actividades y acciones que antes se realizaban. Paralelamente se presenta una sensación de vacío y desesperanza, desaliento y desamparo, apatía y desánimo, como las descritas en la segunda dimensión del IREN-35 (American Psychiatric Association, 2013a).

El tercer factor emergente en esta investigación fue denominado "emociones negativas por percepción de 'amenaza'". En esta dimensión, las principales emociones identificadas fueron la incertidumbre, preocupación, inquietud, sensación de vulnerabilidad, inseguridad, impotencia y fragilidad. Regularmente, la presencia de nerviosismo y tensión persistentes propias de este factor, suelen ir emparejadas de respuestas físicas como tensión muscular, cefaleas, temblores, taquicardias, problemas gastrointestinales, insomnio, entre otros (American Psychiatric Association, 2013b).

A diferencia del segundo factor, en donde predominaron las respuestas de tipo depresivo, esta dimensión se asocia más a respuestas de ansiedad, en donde la inquietud y preocupación por el futuro inmediato se muestran presentes con mayor claridad. La ansiedad -entendida como una sensación de preocupación e intranquilidad-representa el eje articulador de este factor. Como se sabe, la sensación de vulnerabilidad surge de la percepción o avizoramiento de alguna "amenaza" potencial que puede poner en riesgo al individuo. Es decir, la incertidumbre sobre el futuro 
inmediato representa en sí misma una "amenaza", en donde la posibilidad de enfermarse y morir de COVID-19 no necesariamente es una idea extrema o lejana.

Igualmente, a diferencia del primer factor que aludía a respuestas emocionales propias del confinamiento o cuarentena, las respuestas emocionales del resto de las dimensiones particularmente la tercera y cuarta-se centran en la enfermedad en sí misma y en las consecuencias y amenazas que ésta representa. Como bien lo señalan Sandin et al., (2020), ciertos aspectos como la ambigüedad y falta de control durante la pandemia de coronavirus, su carácter impredecible, la letalidad del agente invasor, pueden generar por sí mismas alteraciones psicológicas relacionadas con la percepción de "amenaza" a la propia salud personal.

Justamente esta percepción de "amenaza" identificada en nuestro estudio, articula en un mismo eje a las emociones de incertidumbre, preocupación, inquietud, vulnerabilidad, inseguridad, impotencia, y fragilidad. En su conjunto -y si persisten con el tiempo, a la par de que su intensidad aumente- la presencia de dichas emociones podría coadyuvar en la configuración de trastornos de ansiedad diversos.

El trastorno de ansiedad generalizada (TAG), por ejemplo, está asociado justamente a la presencia de angustia y preocupación excesiva con respecto a ciertos episodios de la vida. Habitualmente, la evolución es fluctuante y crónica y empeora durante periodos de estrés. Como se sabe, además de preocupación excesiva, el TAG se configura con algunos de los siguientes síntomas: agitación o nerviosismo, fatiga, dificultades en la concentración, irritabilidad, tensión muscular y trastornos del sueño (American Psychiatric Association, 2013b). De acuerdo con Mayo Clinic (2020), las preocupaciones y la ansiedad derivadas del COVID-19 pueden resultar abrumadoras y, en conjunto con el distanciamiento social, hacen que sus efectos sean todavía más complejos y desestabilizadores.

Por último, el cuarto factor identificado en esta investigación alude a la presencia de "emociones negativas por 'percepción de peligro intenso"”. En efecto, una sensación de peligro se experimenta ante eventos, experiencias o situaciones interpretados como potencialmente dañinos o perjudiciales para el individuo o la población. En el caso particular de este último factor, aquí se configuran emociones negativas "extremas" derivadas de la pandemia, como pavor, pánico y terror, que no son sino expresiones o manifestaciones extremas del miedo. Lo que distingue a esta última del resto es justamente su intensidad, en donde la experiencia menos fuerte y aguda es el miedo, y las de mayor magnitud el terror, pavor y pánico. 
Como se sabe, el miedo es una emoción básica de sobrevivencia y representa una respuesta normal ante una situación de descontrol y peligro; es una respuesta natural ante una situación real (visible) o imaginaria (invisible) que es percibida como peligrosa y amenazadora para el ser físico o emocional de las personas (Cázares et al., 2019; Gullone \& Lane, 2002; LeDoux, 1999; Talu, 2019). En este sentido, el miedo es esencial y necesario para que los individuos preserven sus vidas (Ekman, 2003; LeDoux, 1999).

De acuerdo con los autores arriba señalados, los miedos pueden dividirse en dos tipos: aquellos basados en una causa concreta, real y visible (como una pandemia); y los que tienen su origen en situaciones abstractas e invisibles. Frente a los primeros, las personas perciben que el peligro es directo y tiene una razón justificable, mientras que ante los segundos se agrega la preocupación de no poder ser capaz de definirlo, y por ello sentirse aterrado por su condición inefable. No obstante, aunque el miedo puede ser justificable dadas las condiciones de peligro real que representa una pandemia, cuando su intensidad se incrementa ostensiblemente, esto da pauta para la configuración de un miedo extremo, que usualmente es denominado terror, pavor o pánico.

En este sentido, lo que en un inicio resultaba ser una respuesta normal y de sobrevivencia -en efecto, el miedo es una emoción básica y normal de sobrevivencia-, con el paso del tiempo y si aumenta su intensidad, puede convertirse en un miedo paralizante y extremo (pavor, pánico o terror), el cual podría terminar por dañar la salud física y mental de las personas. Los componentes emocionales del cuarto factor descrito en esta investigación se derivan justamente de los miedos extremos e intensos: pavor, pánico y terror.

En el caso de la pandemia de COVID-19, si bien es cierto que los riesgos son objetivos y justificables - la posibilidad de contagiarse es real, al igual que su poderosa letalidad-, la percepción de dicho peligro difiere de persona a persona, a partir de las propias valoraciones subjetivas de los riesgos. Si la persona identifica el peligro real (la presencia del coronavirus) y sus posibles consecuencias ("me voy a contagiar o morir"), es probable que el miedo emerja. Pero si el miedo es intenso, derivado de la inmediatez y de la presencia real y objetiva de la enfermedad (en el caso de que algún ser querido o la persona misma se hayan contagiado por el virus), la emoción emergente podría cambiar de intensidad, dando pauta al surgimiento de pavor, pánico o terror.

A medida que va cambiando la intensidad del miedo, la palabra misma también se modifica y adecúa. Si la emoción es moderada y manejable, la palabra adecuada es "miedo", pero si la emoción es muy intensa y persistente, “pavor” o "terror” serán los rótulos más apropiados. En este 
sentido, como señalan Ortony, Clore \& Collins (1996), quizás sea la proximidad subjetiva del acontecimiento (en el caso que nos ocupa la relación directa o indirecta, mediata o inmediata con el virus COVID-19) la que establezca la diferencia.

Los autores arriba citaos señalan que "tendemos a usar palabras como 'miedo' y 'susto' para referirnos a situaciones relativamente 'inminentes', en particular cuando pueden comportar daño corporal, mientras que usamos términos como 'preocupación' y 'aprensión' con relación a posibilidades más remotas y que son amenazas 'posiblemente menos serias"' (Ortony, Clore \& Collins, p. 137, 1996). Resulta claro, en este sentido, que el miedo intenso -es decir, la presencia de pavor, pánico o terror-implicaría la "experiencia directa con el peligro real de la pandemia", en tanto la persona está siendo víctima de la propia enfermedad o testigo directo de un caso cercano.

La percepción de peligro que se encontró en este estudio puede relacionarse con lo reportado en otras investigaciones a nivel mundial. Sandín et al., 2020, por ejemplo, reportaron que la población española presentó miedo al COVID-19 por el posible contagio, enfermedad y muerte que implicaba, así como por la carencia de productos de consumo básicos (bienes de primera necesidad) y por la pérdida de ingresos. Podría considerarse que el riesgo latente de infección y lo que esto implica, genera una percepción de peligro, sobre todo si no hay suficiente confianza en el sistema de salud o en los recursos particulares para atender y financiar los gastos que implica una estancia hospitalaria bajo estas circunstancias (Brooks et al., 2020; Cortés-Álvarez et al., 2020).

La percepción de peligro que se reporta en el presente estudio podría estar relacionada justamente con algunos de los factores previamente descritos. En este sentido, una sensación de peligro se experimenta ante eventos "interpretados" como potencialmente dañinos para el individuo o la población en cuestión. Si bien en México ha existido bastante flujo de información con respecto a la pandemia, se ha percibido cierta ambivalencia con respecto a cómo interpretarla (Cortés-Álvarez et al., 2020). La incertidumbre también puede ser, en este sentido, otro factor que esté mediando la respuesta de la población con respecto a la pandemia por COVID-19 (Williams et al., 2020). El no conocer por cuánto tiempo se llevarán a cabo las medidas de distanciamiento social y cómo será la cotidianidad a mediano y largo plazo, podría estar contribuyendo en la configuración de afectos negativos en las personas.

Así como las emociones constitutivas de los dos factores inmediatamente precedentes (factor 2 y 3 ) estaban relacionadas con una posible configuración de trastornos (particularmente 
del estado de ánimo y de ansiedad), el último factor aquí descrito podría estar vinculado con la emergencia posible del trastorno de estrés postraumático (PTSD), ya que, como se sabe, éste integra respuestas relacionadas con el miedo extremo y la presencia de experiencias traumáticas. De acuerdo con los criterios establecidos en el DSM-V (APA, 2013c), el PTSD es un trastorno que surge como consecuencia de la exposición directa a sucesos traumáticos (como accidentes y epidemias) o a la presencia indirecta de sucesos traumáticos ocurridos a otros (particularmente familiares y seres queridos). Entre los criterios diagnósticos del PTSD se encuentra justamente la presencia de un "estado emocional negativo persistente", como "miedo", "terror" o "culpa" (APA, 2013c).

En suma, los cuatro factores constitutivos del IREN-35 que aquí se presentan, dan cuenta de un conjunto de emociones de carácter perturbador que están asociados, de algún modo u otro, a la exposición directa a la pandemia de COVID-19 y a las consecuencias diversas que ésta produce. La experiencia global del coronavirus, así como la elevada letalidad en muchas partes del mundo, ha generado efectos múltiples en distintas esferas de la vida. En términos de salud mental, resultan cada vez más claros los efectos nocivos de la pandemia, incluida la denomina fatiga pandémica, que es una desmotivación que ha surgido en la población mundial, cansada de cumplir con comportamientos protectores y medidas de seguridad intermitentes (OMS, 2020). Sin ánimos de patologizar a la experiencia subjetiva del COVID-19, estamos convencidos de que los impactos múltiples del coronavirus en el mundo también han permeado a la esfera psicológica, particularmente en lo que respecta al estado de ánimo, cogniciones, comportamientos y emociones de las personas.

Si bien es cierto que la mayor parte de las emociones experimentadas son respuestas normales y de sobrevivencia frente a una situación inédita y no pocas veces traumática -la pandemia de coronavirus lo ha sido sin duda-, también lo es que si este conjunto de emociones perturbadoras se prolongan en el tiempo y aumentan su intensidad, esto podría generar un cúmulo de malestares psicoemocionales diversos, los cuales podrían estar eventualmente asociados, en un futuro, a diversos trastornos mentales de distinto tipo. De ahí la importancia del IREN-35, que fue concebido como un instrumento que puede ayudar en la identificación preventiva de emociones negativas y perturbadoras las cuales - si no se atienden de manera correcta y oportuna- pueden convertirse en elementos perjudiciales y nocivos en la salud mental de las personas. 
Por último, consideramos que existen al menos dos limitaciones importantes en este estudio que podrían ser subsanadas en investigaciones posteriores: en primer término, la limitante de que la muestra aquí empleada se circunscribió únicamente a la Zona Metropolitana de la Ciudad de México (ZMCM), dejando fuera otras ciudades igualmente afectadas por la pandemia. En segundo término, la baja participación de adultos mayores (únicamente el 2.4\% de la muestra utilizada). Consideramos importante ampliar el espectro de aplicación del IREN-35 en próximas investigaciones, con el objeto de corroborar la validez del instrumento en otros entornos geográficos del país y de América Latina.

\section{Agradecimientos}

Agradecemos al Consejo Nacional de Ciencia y Tecnología (CONACYT) por el apoyo y estímulo económico brindado a través del Sistema Nacional de Investigadores.

\section{Referencias}

American Psychiatric Association. (2013a). Trastornos depresivos. En Manual diagnóstico y estadístico de los trastornos mentales $\left(\begin{array}{llll}5 & \text { a } & \text { ed.). }\end{array}\right.$ https://doi.org/10.1176/appi.books.9780890425596

American Psychiatric Association. (2013b). Trastornos de ansiedad. En Manual diagnóstico y $\begin{array}{lllllll}\text { estadístico de } & \text { los } & \text { trastornos } & \text { a } & \text { ed. }) \text {. }\end{array}$ https://doi.org/10.1176/appi.books.9780890425596

American Psychiatric Association. (2013c). Trastornos relacionados con traumas y factores de estrés. En Manual diagnóstico y estadístico de los trastornos mentales (5 a ed.). https://doi.org/10.1176/appi.books.9780890425596

Bei, B., Bryant, C., Gilson, K. M., Koh, J., Gibson, P., Komiti, A., Jackson, H., \& Judd, F. (2013). A prospective study of the impact of floods on the mental and physical health of older adults. Aging and Mental Health, 17(8), 992-1002. https://doi.org/10.1080/13607863.2013.799119

Beutel, M. E., Klein, E. M., Brähler, E., Reiner, I., Jünger, C., Michal, M., Wiltink, J., Wild, P. S., Münzel, T., Lackner, K. J., \& Tibubos, A. N. (2017). Loneliness in the general population: prevalence, determinants and relations to mental health. BMC psychiatry, 17(1), 1-7. https://doi.org/10.1186/s12888-017-1262-x 
Brooks, S. K., Webster, R. K., Smith, L. E., Woodland, L., Wessely, S., Greenberg, N., \& Rubin, G. J. (2020). The psychological impact of quarantine and how to reduce it: rapid review of the evidence. The Lancet, 395, 912-920. https://doi.org/10.1016/S0140-6736(20)30460-8

Calhoun, Ch. \& Solomon, R. (1989). ¿Qué es una emoción? Lecturas clásicas de psicología filosófica. México: Fondo de Cultura Económica.

Cázares, F., Carrillo, D., González, L. A., y Rodríguez, H. E. (2019). Miedo al tratamiento odontológico en escolares mexicanos. Revista Cubana de Estomatología, 56(2), 123 134.http://web.b.ebscohost.com.pbidi.unam.mx:8080/ehost/pdfviewer/pdfviewer?vid=5\& $\underline{\text { sid=12aec835-cf2a-443e-8cd8-1328c794cfe0\%40pdc-v-sessmgr02 }}$

Chóliz, M. (2005). Psicología de la emoción. El proceso emocional. Madrid: Versión en línea. https://www.uv.es/choliz/Proceso\%20emocional.pdf

Clark, D. (2002). Investigación cuantitativa en psicología: Del diseño experimental al reporte de investigación. Oxford Press.

Centro de Investigación y Docencia Económicas. (2020, 11 de mayo). Violencia de Género en tiempos de COVID-19. Recuperado el 4 de julio de 2020, de https://www.cide.edu/coronavirus/2020/05/11/violencia-de-genero-en-tiempos-de-covid$\underline{19 /}$

Cohen, J. (1992). A Power Primer. Psychological Bulletin, 112(1), 155-159.

Cortés-Álvarez, N., Piñeiro-Lamas, R., \& Vuelvas-Olmos, C. (2020). Psychological Effects and Associated Factors of COVID-19 in a Mexican Sample. Disaster Medicine and Public Health Preparedness, 1-12. https://doi.org/10.1017/dmp.2020.215

De la Hoz, G. (2020). Las pérdidas y el duelo en tiempos de COVID-19. Organización Panamericana de la Salud. Recuperado el 4 de julio de 2020, de https://www.paho.org/es/eventos/perdidas-duelo-tiempos-covid-19

Ekman, P. (2003). El rostro de las emociones. Qué nos revelan las expresiones faciales. Barcelona: RBA Bolsillo.

El-Zoghby, S. M., Soltan, E. M., \& Salama, H. M. (2020). Impact of the COVID-19 Pandemic on Mental Health and Social Support among Adult Egyptians. Journal of Community Health, 45, 689-695. https://doi.org/10.1007/s10900-020-00853-5

Flores-Morales, R. (2020a, agosto). Duelo sin réquiem. Edición especial Proceso, 59.

Flores-Morales, R. (2020b, 5 de abril). Miedo, ansiedad, frustración, aburrimiento: Efectos 
colaterales de la pandemia de COVID-19. Revista Proceso.

García, J. M. (2007, Enero-Junio). Las palabras prefijadas con des-. [boletín n. ${ }^{87}$ ]. Boletín de la Real Academia de la Lengua Española. https://www.academia.edu/35685576/Las_palabras_prefijadas_con_des_

Gobierno de México. (2020, 24 de marzo). Anuncia gobierno federal inicio de fase 2 de la epidemia de COVID-19. Recuperado el 4 de julio de 2020, de https://presidente.gob.mx/anuncia-gobierno-federal-inicio-de-fase-2-de-la-epidemia-decovid-19/

González Ramírez LP, Martínez Arriaga RJ, Hernández-Gonzalez MA, \& De la Roca-Chiapas JM, (2020). Psychological Distress and Signs of Post-Traumatic Stress in Response to the COVID-19 Health Emergency in a Mexican Sample. Psychology Research and Behavior Management, 13, 589-597. https://doi.org/10.2147/PRBM.S259563

González-Sanguino, C., Ausín, B., Castellanos, M. Á., Saiz, J., López-Gómez, A., Ugidos, C., \& Muñoz, M. (2020). Mental health consequences during the initial stage of the 2020 Coronavirus pandemic (COVID-19) in Spain. Brain, Behavior, and Immunity, 87, 172-176. https://doi.org/10.1016/j.bbi.2020.05.040

Gullone, E., \& Lane, B. (2002). The Fear Survey Schedule for Children-II: a validity examination across response format and instruction type. Clinical Psychology and Psychotherapy, 9(1), 55-67. https://doi.org/10.1002/cpp.327

Hernández, R., Fernández, C. \& Baptista, P. (2014). Muestreo en la investigación cualitativa. En Metodología de la investigación. México: McGrawHill.

Hao, F., Tan, W., Jiang, L., Zhang, L., Zhao, X., Zou, Y., Hu, Y., Luo, X., Jiang, X., McIntyre, R., Tran, B., Sun, J., Zhang, Z., Ho, R., Ho, C., \& Tam, W. (2020). Do psychiatric patients experience more psychiatric symptoms during COVID-19 pandemic and lockdown? A case-control study with service and research implications for immunopsychiatry. Brain, Behavior, and Immunity, 87, 100-106. https://doi.org/10.1016/j.bbi.2020.04.069

Holt-Lunstad, J., Smith, T. B., Baker, M., Harris, T., \& Stephenson, D. (2015). Loneliness and Social Isolation as Risk Factors for Mortality: A Meta-Analytic Review. Perspectives on Psychological Science, 10(2), 227-237. https://doi.org/10.1177/1745691614568352

James, W. (1890). The Principles of Psychology. Una traducción de esta obra está disponible en: James, W. (1947). Principios de Psicología. Buenos Aires: EMECE Editores. 
Kerlinger, F. \& Lee, H. (2002). Investigación del comportamiento. Métodos de investigación en ciencias sociales. McGraw-Hill.

LaJoie, A. S., Sprang, G., \& McKinney, W. P. (2010). Long-term effects of Hurricane Katrina on the psychological well-being of evacuees. Disasters, 34(4), 1031-1044. https://doi.org/10.1111/j.1467-7717.2010.01181.x

Le Breton, D. (2013). Por una antropología de las emociones. Revista Latinoamericana de Estudios sobre Cuerpos, Emociones y $\quad$ Sociedad. $10 \quad$ (4), http://www.relaces.com.ar/index.php/relaces/article/viewFile/208/145

LeDoux, J. (1999). La influencia de las emociones. En Conlan, R (1ed.), Estados de ánimo. Cómo nuestro cerebro nos hace ser como somos (pp. 119-141). Barcelona: Paidós Transiciones.

Lewis, C., \& Short, C. (1879). A Latin Dictionary, (1ed.). Oxford: Clarendon Press.

Luchetti, M., Lee, J. H., Aschwanden, D., Sesker, A., Strickhouser, J. E., Terracciano, A., \& Sutin, A. R. (2020). The Trajectory of Loneliness in Response to COVID-19. American Psychologist. 1-12. http://dx.doi.org/10.1037/amp0000690

Marina, J.A. (1996). El laberinto sentimental. Barcelona: Anagrama.

Martínez, F. (2020, 25 de abril). COVID-19: Llamadas por violencia doméstica superan las 26 mil en marzo. La Jornada. https://www.jornada.com.mx/ultimas/sociedad/2020/04/25/covid19-llamadas-por-violencia-domestica-superan-las-26-mil-en-marzo-796.html

Mayo Clinic. (2020, 11 de marzo). Is worry about COVID-19 disrupting your life? Recuperado el 4 de julio de 2020, de https://newsnetwork.mayoclinic.org/discussion/is-worry-aboutcovid-19-disrupting-your-life/

McGinty, E., Presskreischer, R., Han, H., \& Barry, L. (2020). Psychological Distress and Loneliness Reported by US Adults in 2018 and April 2020. JAMA. 324(1), 93-94. https://doi:10.1001/jama.2020.9740

Milman, E., Lee, S. A., \& Neimeyer, R. A. (2020). Social isolation as a means of reducing dysfunctional coronavirus anxiety and increasing psychoneuroimmunity. Brain, Behavior, and Immunity, 87, 138-139. https://doi.org/10.1016/j.bbi.2020.05.007

Moccia, L., Janiri, D., Pepe, M., Dattoli, L., Molinaro, M., De Martin, V., Chieffo, D., Janiri, L., Fiorillo, A., Sani, Gabriele, \& Di Nicola, M. (2020). Affective temperament, attachment style, and the psychological impact of the COVID-19 outbreak: an early report on the Italian general population. Brain, Behavior, and Immunity, 87, 75-79. 
https://doi.org/10.1016/j.bbi.2020.04.048

Nagamine, M., Yamamoto, T., Shigemura, J., Tanichi, M., Yoshino, A., Suzuki, G., Yoshitomo, T., Miyazaki, M., Uwabe, Y., Harada, N., \& Shimizu, K. (2018). The Psychological Impact of the Great East Japan Earthquake on Japan Ground Self-Defense Force Personnel: A Three-Wave, One-Year Longitudinal Study. Psychiatry, 81(3), 288-296. https://doi.org/10.1080/00332747.2017.1333340

National Academies of Sciences, Engineering, and Medicine (2020). Social Isolation and Loneliness in Older Adults: Opportunities for the Health Care System. Washington, DC: The National Academies Press. https://doi.org/10.17226/25663.

Trad N. K., Wharam, J. F., \& Druss, B. (2020). Addressing Loneliness in the Era of COVID-19. JAMA Health Forum. Published online June 1, 2020. doi:10.1001/jamahealthforum.2020.0631

Nicol, G. E., Piccirillo, J. F., Mulsant, B. H., \& Lenze, E. J. (2020). Action at a Distance: Geriatric Research during a Pandemic. Journal of the American Geriatrics Society, 68(5), 922-925. https://doi.org/10.1111/jgs.16443

Nunnally, J. \& Berstein, I. (1994). Psychometric theory (3rd ed.). New York: McGraw-Hill.

Organización de las Naciones Unidas. (2020, abril). COVID-19 y su impacto en la violencia contra las mujeres $y$ niñas. Recuperado el 4 de julio de 2020, de https://www2.unwomen.org/media/field\%20office\%20mexico/documentos/publicaciones/ 2020/abril\%202020/covid19_violenciamujeresninas_generalabril2020.pdf?la=es\&vs=245 $\underline{7}$

Organización Mundial de la Salud. (2021). Coronavirus disease (COVID-19) Weekly Epidemiological Update and Weekly Operational Update. Consultado el 28 de enero de 2021. https://www.who.int/publications/m/item/weekly-epidemiological-update---27january-2021

Orellana, C. I., \& Orellana, L. M. (2020). Predictores de síntomas emocionales durante la cuarentena domiciliar por pandemia de COVID-19 en El Salvador. Actualidades en Psicología, 34(128), 103-120. https://doi.org/10.15517/ap.v34i128.41431

Ortony, A., Clore, G. \& Collins, A. (1996). La estructura cognitiva de las emociones. Siglo XXI. Parrado-González, A., \& León-Jariego, J. (2020). COVID-19: Factores asociados al malestar emocional y morbilidad psíquica en población española. Rev Esp Salud Pública, 94, 1-16. 
Priego-Parra, B., Triana-Romero, A., Pinto-Gálvez, S., Durán, C., Salas-Nolasco, O., Manríquez, M., Ramos-de-la-Medina, A., \& Remes-Troche, J. M. (2020). Anxiety, depression, attitudes, and internet addiction during the initial phase of the 2019 coronavirus disease (COVID-19) epidemic: A cross-sectional study in México. MedRxiv. https://doi.org/10.1101/2020.05.10.20095844

Real Clear Politics (2021). Coronavirus (COVID-19) Global deaths. Consultado el 28 de enero de 2021. https://www.realclearpolitics.com/coronavirus/

Reuters (2021, 28 de enero). Estadísticas diarias de COVID-19 en América Latina y el Caribe. Consultado el 28 de enero de 2021, de https://graphics.reuters.com/world-coronavirustracker-and-maps/es/regions/latin-america-and-the-caribbean/

Reyes-Lagunes, I. \& García, L.F. (2008). Procedimiento de validación psicométrica culturalmente relevante: un ejemplo. En La psicología social en México V. XII (pp. 625-630). México: Amepso.

Rottenberg, J., \& Gross, J. (2007). Emotion and Emotion Regulation: A Map for Psychotherapy Researchers. Clinical Psychology: Science and Practice, 14(4), 323-328. https://doi.org/10.1111/j.1468-2850.2007.00093.x

Samaniego, A., Urzúa, A., Buenahora, M., \& Vera-Villarroel, P. (2020). Sintomatología asociada a Trastornos de Salud Mental en trabajadores sanitarios en Paraguay: Efecto COVID-19. Revista Interamericana de Psicología, 54(1), 1-19. https://10.30849/ripijp.v54i1.1298

Sandín, B., Valiente, R. M., García-Escalera, J., \& Chorot, P. (2020). Impacto psicológico de la pandemia de COVID-19: Efectos negativos y positivos en población española asociados al periodo de confinamiento nacional. Revista de Psicopatología y Psicología Clínica, 25(1), 1-22. https://doi.org/10.5944/rppc. 27569

Shalev, D., \& Shapiro, P. A. (2020). Epidemic psychiatry: The opportunities and challenges of COVID-19. General Hospital Psychiatry, 64, 68-71.

Secretaría de Salud (2020). COVID-19 México: información general. Recuperado el 4 de julio de 2020, de https://coronavirus.gob.mx/datos/

Solís-Cámara, P., Meda, R., Moreno, B., \& Juárez, P. (2016). Estructura factorial del Cuestionario de Salud General GHQ-12 en población general de México. Salud \& Sociedad, 7, 62-76. DOI: $10.22199 / \mathrm{S} 07187475.2016 .0001 .00004$

Somma, A., Gialdi, G., Krueger, R. F., Markon, K. E., Frau, C., Lovallo, S., \& Fossati, A. (2020). 
Dysfunctional personality features, non-scientifically supported causal beliefs, and emotional problems during the first month of the COVID-19 pandemic in Italy. Personality and Individual Differences, 165, 1-6. https://doi.org/10.1016/j.paid.2020.110139

Stratta, P., Capanna, C., Riccardi, I., Perugi, G., Toni, C., Dell'Osso, L., \& Rossi, A. (2013). Spirituality and Religiosity in the Aftermath of a Natural Catastrophe in Italy. Journal of Religion and Health, 52, 1029-1037. https://doi.org/10.1007/s10943-012-9591-z

Talu, E. (2019). Reflections of Fears of Children to Drawings. European Journal of Educational Research, 8(3), 763-779. https://doi.org/10.12973/eu-jer.8.3.763

Tan, W., Hao, F., McIntyre, R. S., Jiang, L., Jiang, X., Zhang, L., Zhao, X., Zou, Y., Hu, Y., Luo, X., Zhang, Z., Lai, A., Ho, R., Tran, B., Ho, C, \& Tam, W. (2020). Is returning to work during the COVID-19 pandemic stressful? A study on immediate mental health status and psychoneuroimmunity prevention measures of Chinese workforce. Brain, Behavior, and Immunity, 87, 84-92. https://doi.org/10.1016/j.bbi.2020.04.055

Trad, N, K., Wharam, F., \& Druss, B. (2020). Addressing Loneliness in the Era of COVID-19. Recuperado el 4 de julio de 2020, de https://jamanetwork.com/channels/healthforum/fullarticle/2766811

Varshney, M., Parel, J. T., Raizada, N., \& Sarin, S. K. (2020). Initial psychological impact of COVID-19 and its correlates in Indian Community: An online (FEEL-COVID) survey. PLoS ONE, 15(5), 1-11. https://doi.org/10.1371/journal.pone.0233874

Wang, C., Pan, R., Wan, X., Tan, Y., Xu, L., McIntyre, R. S., Choo, F. N., Tran, B., Ho, R., Sharma, V. K., \& Ho, C. (2020). A longitudinal study on the mental health of general population during the COVID-19 epidemic in China. Brain, Behavior, and Immunity, 87, 40-48. https://doi.org/10.1016/j.bbi.2020.04.028

Ward, M., McGarrigle, Ch., Hever, A., O’Mahoney, P., Moynihan,S., Loughran, G., \& Kenny, R.A. (2020). Loneliness and social isolation in the COVID-19 Pandemic among the over 70s: Data from The Irish Longitudinal Study on Ageing (TILDA) and ALONE. TILDA. https://www.doi.org/10.38018/TildaRe.2020-07.

Williams, S. N., Armitage, C. J., Tampe, T., \& Dienes, K. (2020). Public perceptions and experiences of social distancing and social isolation during the COVID-19 pandemic: A $\begin{array}{llllll}\text { UK-based focus group study. BMJ OPEN, } & 27, & 1-8 .\end{array}$ https://doi.org/10.1101/2020.04.10.20061267 